This is an electronic reprint of the original article. This reprint may differ from the original in pagination and typographic detail.

Author(s): Miettinen, Arttu; Ekman, Axel; Chinga-Carrasco, Gary; Kataja, Markku

Title: Measuring intrinsic thickness of rough membranes: application to nanofibrillated cellulose films

Year: $\quad 2015$

Version:

Please cite the original version:

Miettinen, A., Ekman, A., Chinga-Carrasco, G., \& Kataja, M. (2015). Measuring intrinsic thickness of rough membranes: application to nanofibrillated cellulose films. Journal of Materials Science, 50(21), 6926-6934. https://doi.org/10.1007/s10853015-9243-2

All material supplied via JYX is protected by copyright and other intellectual property rights, and duplication or sale of all or part of any of the repository collections is not permitted, except that material may be duplicated by you for your research use or educational purposes in electronic or print form. You must obtain permission for any other use. Electronic or print copies may not be offered, whether for sale or otherwise to anyone who is not an authorised user. 


\title{
Measuring intrinsic thickness of rough membranes: Application to nanofibrillated cellulose films
}

\author{
Arttu Miettinen*1, Axel Ekman ${ }^{\dagger 1}$, Gary Chinga-Carrasco ${ }^{\ddagger 2}$ and Markku Kataja ${ }^{\S 1}$ \\ ${ }^{1}$ University of Jyvaskyla, Department of Physics, P.O. Box 35 (YFL) FI-40014 Jyvaskyla, Finland \\ ${ }^{2}$ Paper and Fibre Research Institute (PFI), Høgskoleringen 6 B, NO-7491 Trondheim, Norway
}

Journal of Materials Science 50(21), doi: 10.1007/s10853-015-9243-2

\begin{abstract}
Adequate measurement of thickness of sheet-like materials or membranes is most important for quantifying their properties such as density, barrier properties and mechanical strength. Depending on the surface roughness of the membrane, the thickness measured by standard micrometer devices (apparent thickness) may considerably overestimate the actual geometrical mean thickness (intrinsic thickness) required for such purposes. In this work, we present a method for correcting the measured apparent thickness value of thin membranes for their surface roughness, thereby obtaining an improved estimate of the intrinsic thickness. The surface roughness data required for the correction can be obtained by common surface profiling techniques. The method includes a calibration parameter, the value of which can be found experimentally by independent measurements, or can be estimated theoretically using results from standard mechanical contact theory.
\end{abstract}

\footnotetext{
*arttu.miettinen@phys.jyu.fi, Tel.: +358-408054102

†axel.ekman@jyu.fi

$\ddagger$ gary.chinga.carrasco@pfi.no

§markku.kataja@jyu.fi
}

The method is tested on a set of nanofibrillated cellulose films with varying roughness levels controlled by pulp fibre content. The surface topography of film samples was measured using laser profilometry and the method was calibrated experimentally using data from X-ray microtomographic images for one type of film. The intrinsic thickness estimates given by the new method are generally in good accordance with independent results obtained from $\mathrm{X}$-ray microtomography.

Keywords: Thickness, Roughness, X-ray tomography, Nanofibrillated cellulose, NFC

\section{Introduction}

The thickness of a rough sheet material or membrane, e.g., nanofibrillated cellulose film, paper, or rubber plate, is traditionally measured by a micrometer following the standard ISO534 [1]. The method is based on pressing the membrane between two smooth steel plates with a predetermined force and recording the distance between the plates. The result of this measurement is commonly referred to as the 
apparent thickness while the term intrinsic thickness (also called effective thickness) is used to denote the mean of the local thickness distribution. The intrinsic thickness can be approximated using, e.g., micrometer with soft rubber plates, mercury immersion method or two-sided profilometry. A review of various methods and their limitations can be found e.g. in [2].

For membranes with the same intrinsic thickness, the apparent thickness tends to increase with roughness. Generally, this happens when only a small number of contacts between the highest peaks on a rough surface and the pressing plates can carry the total compressing load used in the apparent thickness measurement. Recently, it was demonstrated that the roughness of cellulose nanopapers has a major influence on the apparent thickness [3]. Overestimation of the thickness of the film may, in turn, lead to underestimation of important bulk properties such as density, tensile strength and gas barrier characteristics (see e.g. [4-7]).

The topography of a given surface can be quantified using various techniques, e.g., Parker Print Surf (PPS), atomic force microscopy (AFM), white light interferometry, stylus and laser profilometry, confocal laser scanning microscopy (CLSM) and scanning electron microscopy (SEM) (see e.g. [8-10]). Laser profilometry is a widely used technique for assessing the roughness of many types of sheet materials and membranes. The method can assess relatively large areas (in centimeter scale), and with suitable lateral and z-resolution, typically of the order of $1 \mu \mathrm{m}$ and $10 \mathrm{~nm}$, respectively. The thickness and the surface roughness of a membrane sample can be independently found using $\mathrm{X}$-ray microtomography (X$\mu \mathrm{CT})$. The method yields a three-dimensional volume image of the sample where contrast is based on the local X-ray absorption coefficient [11]. Based on the image it is possible to estimate the local thickness and the surface topography of both sides of the sample with good resolution. A disadvantage of the method is that it is relatively time-consuming and expensive as compared to micrometer and profilometer measurements.

In this study we propose a method for correcting the results from the micrometric apparent thickness measurements thereby finding estimates for the intrinsic thickness of rough membranes. The correction is based on measuring the surface roughness of the membrane using any standard laboratory equipment with appropriate resolution and measuring area. We consider two limiting cases related to the assumed behaviour of the membrane under compression in micrometric thickness measurement, the inflexible case and the freely flexible case. This leads to two different correction formulas, both including a material-dependent coefficient which remains as a free calibration parameter of the method. The method is tested on a set of nanofibrillated cellulose (NFC) film samples with varying degree of roughness, measured here using laser profilometry. The value of the calibration parameter is estimated both theoretically using a correlation formula and elastic material properties found in the literature, and experimentally utilizing X-ray microtomographic techniques on a single calibration sample. The intrinsic thickness estimates thereby obtained are compared with those from independent three-dimensional analysis of X-ray microtomographic images.

\section{Estimation of intrinsic thickness based on apparent thickness and surface topography}

Referring to the qualitative cross-sectional geometry of a rough membrane shown in Figure 1, we express the local thickness $d(x)$ of the membrane at position 


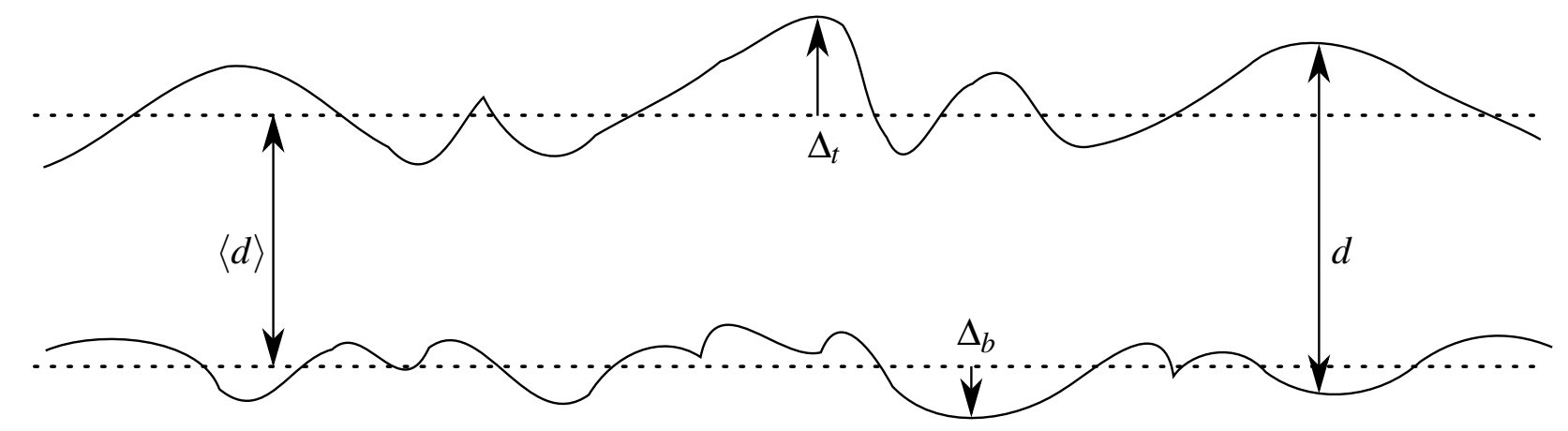

Figure 1: Schematic representation of the cross-sectional structure of a film. The solid lines represent film surfaces and the dashed lines represent average positions of surfaces. Arrows corresponding to top and bottom roughness, $\Delta_{t}$ and $\Delta_{b}$, are drawn towards their positive directions.

$x \in \mathbb{R}^{2}$ in a form

$$
d(x)=\langle d\rangle+\Delta_{\mathrm{t}}(x)+\Delta_{\mathrm{b}}(x) .
$$

Here $\langle d\rangle$ is the intrinsic thickness of the membrane (i.e. the mean thickness over membrane area), and $\Delta_{\mathrm{t}}(x)$ and $\Delta_{\mathrm{b}}(x)$ are the local height of the top and bottom surfaces, respectively, measured from their average positions.

When applying a micrometer device in measuring the thickness, the membrane sample is compressed between rigid flat parallel plates of total area $A_{0}$. The force applied on the plates is small enough such that no significant overall compression of the membrane occurs. Depending on the material properties and roughness of the surfaces, the plates may be in contact only with isolated peaks on the membrane surfaces. Small scale deformation of membrane material takes place near the contact points, and the total contact area $A_{\mathrm{c}} \ll A_{0}$. Consequently, the distance between the plates, i.e., the measured apparent thickness $d_{\text {app }}$ may differ from the intrinsic thickness $\langle d\rangle$, typically such that $\left.d_{\mathrm{app}}\right\rangle\langle d\rangle$. The objective here is to find a correction $\varepsilon$ to the apparent thickness so as to obtain the intrinsic thickness in a form

$$
\langle d\rangle=d_{\text {app }}-\varepsilon .
$$

In addition to material properties and the surface topography of the membrane sample, the result of the apparent thickness measurement may be affected by local bending of the sample in the measurement condition. Such bending can, in principle, take place in subregions where the sample is in contact with only one of the two compressing plates. Evaluating the effects of such bending seems quite intricate, in general. It appears, however, that two limiting cases, namely the case of an inflexible sample and the case of a freely flexible sample can be analysed in a relatively straightforward manner. Below, we derive the required correction terms for the inflexible case and for the freely flexible case separately.

\subsection{Inflexible case}

By the inflexible case, we refer here to a situation where the local bending of the membrane sample can be neglected in apparent thickness measurement such that, e.g., the geometric center plane of the original (unloaded) sample remains planar when compressed between the plates. Such an assumption is appropriate for samples for which the lateral size scale of surface roughness is small enough so that the 
typical distance between neighbouring contact points on both sides of the sample is small as compared to membrane thickness. In this case, the small scale deformation and the contact area between the sample and the compressing plates on the two sides are independent. Consequently, the contribution to the thickness correction $\varepsilon$ can be obtained independently for the two sides, and is given as

$$
\varepsilon=\varepsilon_{\mathrm{t}}+\varepsilon_{\mathrm{b}},
$$

where $\varepsilon_{\mathrm{t}}$ and $\varepsilon_{\mathrm{b}}$ are the distances between the mean surface position and the plate position for the top and bottom sides, respectively (both taken to be positive in the case that the mean surface position is located between the plate position and the center plane of the membrane).

In order to find the thickness correction for each of the two sides, we assume that the height distribution function of surface area

$$
p_{i}(\Delta)=\frac{1}{A_{0}} \frac{d A_{i}}{d \Delta}, \quad i=\mathrm{t}, \mathrm{b}
$$

is known for both sides of the sample (see Sect. 3). Here, $A_{i}$ denotes the area of surface $i$, and $\Delta$ is a surface height variable referred to the mean position of the surface. Assuming further that the compressing force used in the apparent thickness measurement conditions is small enough such that the deformations at the contact locations are small, the relative contact area can be written as

$$
\begin{aligned}
A_{i}^{\mathrm{rel}} & =\frac{1}{A_{0}} \int_{\Delta_{i}(x) \geq \varepsilon_{i}} d A \\
& =\int_{\varepsilon_{i}}^{\infty} p_{i}(\Delta) d \Delta \equiv P_{i}\left(\varepsilon_{i}\right), \quad i=\mathrm{t}, \mathrm{b},
\end{aligned}
$$

where $P_{i}$ is the complementary cumulative surface height distribution function.

On the other hand, based on multiple treatments (e.g., [12-17]), for randomly rough surface, the contact area between a flat plate and a rough surface is proportional to the applied force and inversely proportional to the root-mean-square of the surface slope. As the force used in the apparent thickness measurement is a device-dependent constant, the relative contact area at the final plate position can be written as

$$
A_{i}^{\mathrm{rel}}=\frac{k}{\sigma_{i}^{\mathrm{rms}}}, \quad i=\mathrm{t}, \mathrm{b},
$$

where $k$ is a constant that depends on the measuring force and the properties of the material, and

$$
\sigma_{i}^{\mathrm{rms}}=\sqrt{\left\langle\left|\operatorname{grad}\left(\Delta_{i}\right)\right|^{2}\right\rangle}, \quad i=\mathrm{t}, \mathrm{b},
$$

is the root-mean-square of the surface slope. Here, $\langle\cdot\rangle$ denotes average over the horizontal sample area $A_{0}$. Using Equation 6 we thus get

$$
P_{i}\left(\varepsilon_{i}\right)=\frac{k}{\sigma_{i}^{\mathrm{rms}}}, \quad i=\mathrm{t}, \mathrm{b} .
$$

Inverting this equation yields the surface-specific thickness corrections in the form

$$
\varepsilon_{i}=P_{i}^{-1}\left(\frac{k}{\sigma_{i}^{\mathrm{rms}}}\right), \quad i=\mathrm{t}, \mathrm{b} .
$$

Given the independent contributions from the two surfaces by Equation 10, the total correction for the inflexible case is given by Equation 3, and the estimate for the intrinsic thickness by Equation 2 .

\subsection{Flexible case}

By the flexible case, we refer here to a situation where the membrane sample is able to bend such that significant contact force between the plates and the sample occurs only where the points of contact coincide at the opposite sides of the sample. Such an assumption is appropriate for thin and coarse membranes when the typical distance between contact points is large as compared to membrane thickness. 


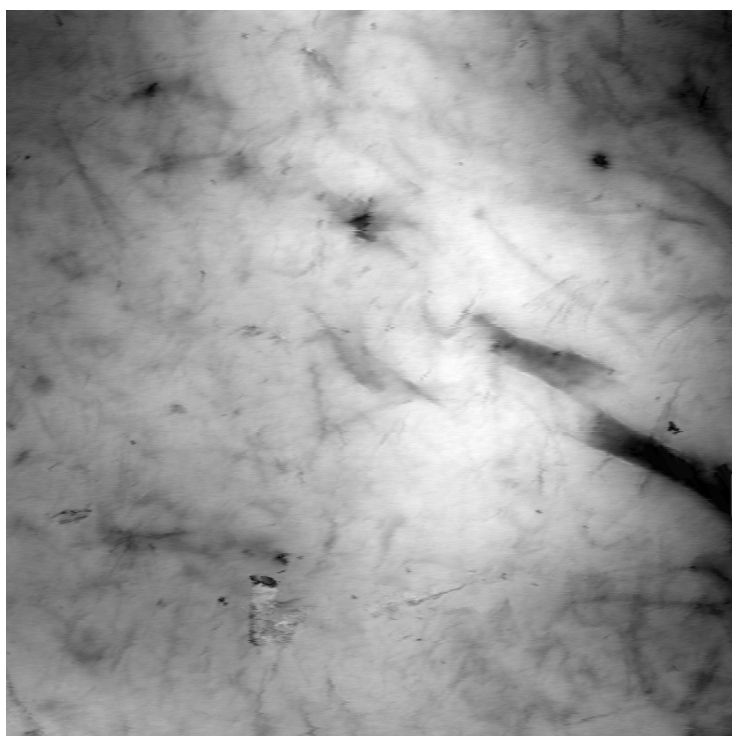

(a)

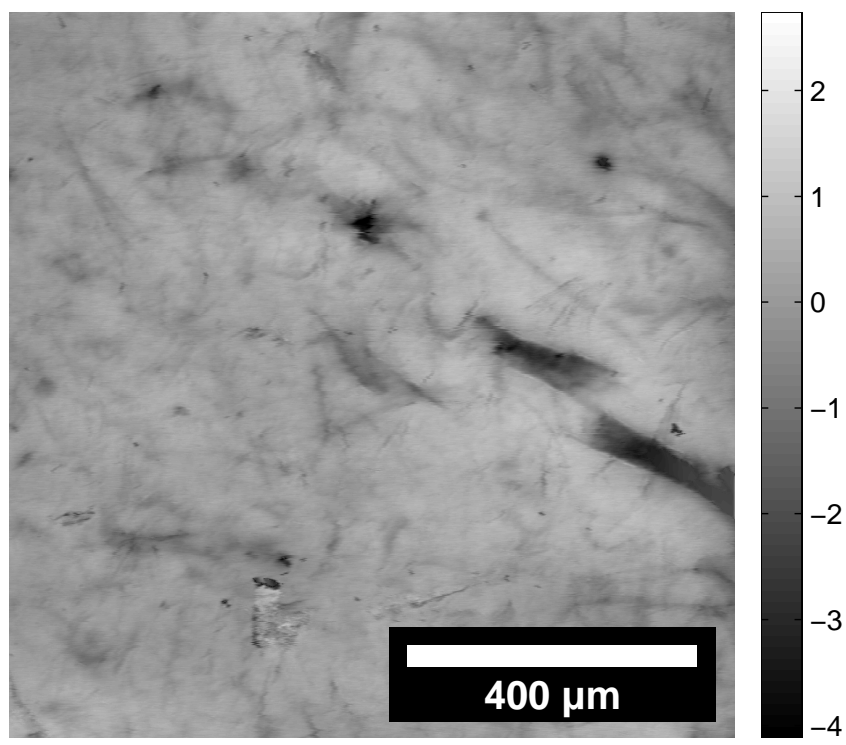

(b)

Figure 2: (a) The original surface map of sample T100 (see below) obtained by laser profilometry and (b) the optimally high-pass filtered map. Values in the color bar are given in micrometers, zero corresponding to the mean of both surface maps. The effect of global curvature evident in (a) is not present in (b). 
In order to analyse such a limiting case, we consider an equivalent sample with one side taken to be even and the other surface rough with surface height given as the sum of the local heights of the top and bottom surfaces of the actual sample as

$$
\Delta_{\mathrm{d}}(x)=\Delta_{\mathrm{t}}(x)+\Delta_{\mathrm{b}}(x) .
$$

According to Equation $1, \Delta_{\mathrm{d}}(x)$ equals the local thickness variation $d(x)-\langle d\rangle$. The thickness correction of the flexible sample can now be calculated analogously with the correction due to one of the sides of an inflexible sample discussed above, but considering a sample with one-sided roughness characterized by the thickness variation $\Delta_{\mathrm{d}}(x)$. It thus remains to find the thickness distribution function of surface area $p_{\mathrm{d}}$ and the root-mean-square of the slope, $\sigma_{\mathrm{d}}^{\text {rms }}$, defined analogously with Equations 4 and 8 , respectively, but replacing the surface height $\Delta_{i}(x)$ by the thickness variation $\Delta_{\mathrm{d}}(x)$.

A straightforward approach would be to measure the surface heights $\Delta_{\mathrm{t}}(x)$ and $\Delta_{\mathrm{b}}(x)$ for the top and bottom side at exactly the same locations $x$ on opposite sides of the membrane such that they can be directly added to get $\Delta_{\mathrm{d}}(x)$, which would then yield $p_{\mathrm{d}}$. It appears, however, that in the present case, it is not necessary to determine $\Delta_{\mathrm{d}}(x)$ explicitly in order to find the required thickness correction. Instead, we assume that the surface height functions $\Delta_{t}(x)$ and $\Delta_{\mathrm{b}}(x)$ are uncorrelated and measured at random locations of the top and bottom sides of the sample. The distribution function $p_{\mathrm{d}}$, being related to the sum of these two independent variables, is thus given by convolution of the distribution functions for the top and bottom surfaces, i.e. $p_{\mathrm{d}}(\Delta)=\left(p_{\mathrm{t}} * p_{\mathrm{b}}\right)(\Delta)$. Furthermore, it is straightforward to verify that in the case of uncorrelated measurements of top and bottom surface shapes, the root-mean-square of the slope of the surface profile $\Delta_{\mathrm{d}}(x)$ is given by

$$
\sigma_{\mathrm{d}}^{\mathrm{rms}}=\sqrt{\left(\sigma_{\mathrm{t}}^{\mathrm{rms}}\right)^{2}+\left(\sigma_{\mathrm{b}}^{\mathrm{rms}}\right)^{2}} .
$$

Defining the complementary cumulative thickness distribution function of surface area by

$$
P_{\mathrm{d}}(\varepsilon)=\int_{\varepsilon}^{\infty}\left(p_{\mathrm{t}} * p_{\mathrm{b}}\right)(\Delta) d \Delta,
$$

the thickness correction for the flexible case is given by

$$
\varepsilon_{\mathrm{d}}=P_{\mathrm{d}}^{-1}\left(\frac{k}{\sigma_{\mathrm{d}}^{\mathrm{rms}}}\right),
$$

and the corresponding estimate for the intrinsic thickness by Equation 2 .

\section{Surface height distributions and calibration of the method}

We now turn to finding the surface height distributions $p_{i}(\Delta)$, the root-mean-square surface slopes $\sigma_{i}^{\text {rms }}$ and the calibration parameter $k$ assuming the apparent thickness $d_{\text {app }}$ and the surface maps $\Delta_{i}(x)$ for top and bottom surfaces to be known. In principle, the surface maps used to evaluate the thickness correction should be determined from a globally flat sample. In practise, the effects of moderate global curvature that may be present in the physical samples, are best removed by high-pass filtering the original surface maps with an appropriate selection of filtering threshold wavelength.

Figure 2a shows an example of an original surface height map obtained by laser profilometry for a nanofibrillated cellulose film sample. In Figure 3 the standard deviation of a high-pass filtered surface height profile is plotted as a function of the filter threshold. The high slope part of the curve at small threshold values is related to the global curvature of the sample (large spatial scales) while the low slope part at high threshold values arises from the small scale roughness relevant for the present purpose. A natural choice of the threshold value for removing global curvature with minimal effect on roughness 


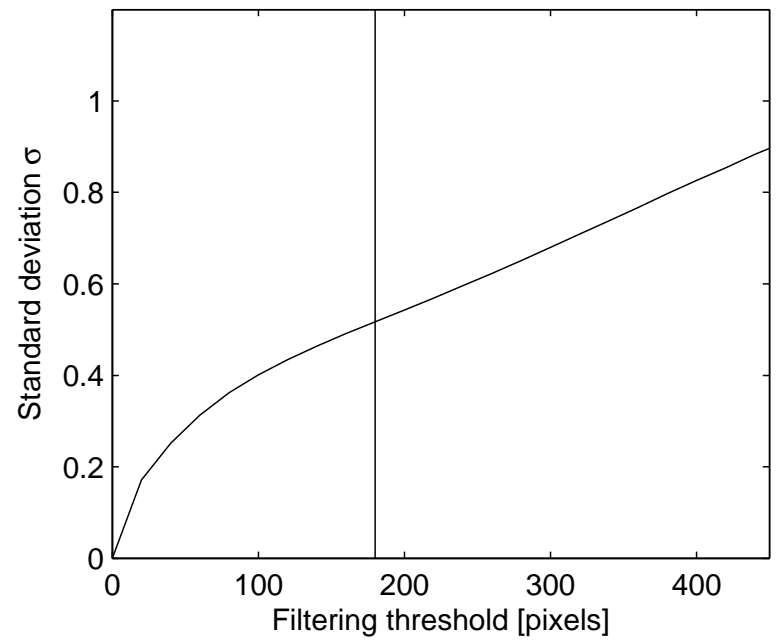

Figure 3: Standard deviation of surface position as a function of high pass filter threshold wavelength for surface height map shown in Figure 2a. The solid vertical line indicates the threshold value at the point of cross-over from large scale to small scale behaviour, i.e. the value of filtering threshold where the derivative of the standard deviation becomes approximately constant. Here, the selected threshold value is the smallest value of filtering threshold $t$ where $d \sigma / d t$ is nondecreasing.

Table 1: Values of root-mean-square roughness $\Delta^{\mathrm{rms}}$ and root-mean-square surface slope $\sigma^{\mathrm{rms}}$ of the top (subscript t) and bottom (subscript b) surfaces of the film types used in the test case.

\begin{tabular}{cccccc}
\hline & T0 & T30 & T50 & T70 & T100 \\
\hline$\Delta_{\mathrm{t}}^{\mathrm{rms}}[\mu \mathrm{m}]$ & $4.8 \pm 0.2$ & $3.9 \pm 0.2$ & $3.0 \pm 0.2$ & $2.4 \pm 0.2$ & $0.55 \pm 0.05$ \\
$\Delta_{\mathrm{b}}^{\mathrm{rms}}[\mu \mathrm{m}]$ & $0.7 \pm 0.2$ & $0.88 \pm 0.06$ & $0.98 \pm 0.07$ & $0.83 \pm 0.10$ & $0.11 \pm 0.02$ \\
$\sigma_{\mathrm{t}}^{\mathrm{rms}}$ & $0.55 \pm 0.02$ & $0.51 \pm 0.02$ & $0.36 \pm 0.02$ & $0.26 \pm 0.02$ & $0.060 \pm 0.002$ \\
$\sigma_{\mathrm{b}}^{\text {rms }}$ & $0.12 \pm 0.01$ & $0.21 \pm 0.02$ & $0.15 \pm 0.01$ & $0.14 \pm 0.01$ & $0.031 \pm 0.002$ \\
\hline
\end{tabular}


is thus at the point of cross-over from large to small scale behaviour, indicated by a solid vertical line in Figure 3. It has been verified that the filtered surface map and the final calculated thickness values show only weak dependence on the threshold value. In the test case discussed below, varying the threshold value by $50 \%$ from its optimal value leads to variation less than $20 \%$ in the calculated intrinsic thickness value. Figure $2 b$ shows the optimally filtered surface map corresponding to the original measured surface map shown in Figure 2a.

The surface maps of the top and bottom surfaces, filtered with the optimal choice of the threshold value as discussed above, are used to compute the rootmean-square surface slope values $\sigma_{i}^{\text {rms }}$ according to Equations 8 and 12 by finite differences. The height distribution functions $p_{i}(\Delta)$, defined by Equation 4 , are obtained from the same surface maps by a straightforward binning. The complementary cumulative distributions $P_{\mathrm{i}}(\varepsilon)$ for the flexible and inflexible cases are finally obtained from Equations 6 and 13, respectively (see Figure 4 below).

The calibration parameter $k$ can be determined experimentally by using some independent method such as $\mathrm{X}-\mu \mathrm{CT}$. Based on a three-dimensional $\mathrm{X}$ $\mu \mathrm{CT}$ image of the sample material, it is straightforward to find the geometrical mean thickness $\langle d\rangle_{\mathrm{CT}}$ by considering the borders between the sample material and the ambient air in the tomographic image. Substituting $\langle d\rangle$ for $\langle d\rangle_{\mathrm{CT}}$ in Equation 2 with the correction $\varepsilon$ defined by Equations 10 or 14 yields the value of $k$ for inflexible and flexible case, respectively. Similar calibration could be done using other techniques such as electron microscopy of membrane cross-sections.

The value of the calibration parameter can also be estimated theoretically. Assuming that the compressing plates used in the apparent thickness measurement are very stiff and smooth compared to the membrane surfaces and that the surface roughness of the membrane consists of spherical asperities with constant radius and constant mechanical properties, the theoretical results derived by Greenwood [13] can be applied, to find that

$$
k=\kappa \frac{F}{A_{0}} \frac{\left(1-v^{2}\right)}{E} .
$$

Here $F$ is the compressing force, and $E$ and $v$ are the Young's modulus and Poisson's ratio of the material, respectively. The constant $\kappa$ is dimensionless and its exact value depends on the distribution of asperity heights. For exponentially and normally distributed asperity height the value is approximately $\kappa \approx 2$. A result similar to Equation 15 can be derived also from the work of Persson $[15,16]$.

The basic assumption leading to Equation 7, and underlying the present method, is that the contact between the membrane and the pressing plate can be described by elastic Herzian theory. This, in turn, requires that the two materials can be considered as elastic half-infinite bodies with constant elastic material properties at contact points. These assumptions may be violated, e.g., by possible irregular surface structures of the membrane such as protruding long filaments or lamellae as well as by large pores near the surface which, nevertheless, contribute to results from surface profilometer. The method may thus not be directly applicable to materials with such surface irregularities. These may include, e.g., certain paper grades, non-woven materials and very porous membranes. At the least, with such materials, special attention should be paid in calibration and in interpretation of profilometer data.

\section{Results}

The method discussed above was applied in estimating the intrinsic thickness of nanofibrillated cellulose films manufactured from never dried Pinus 


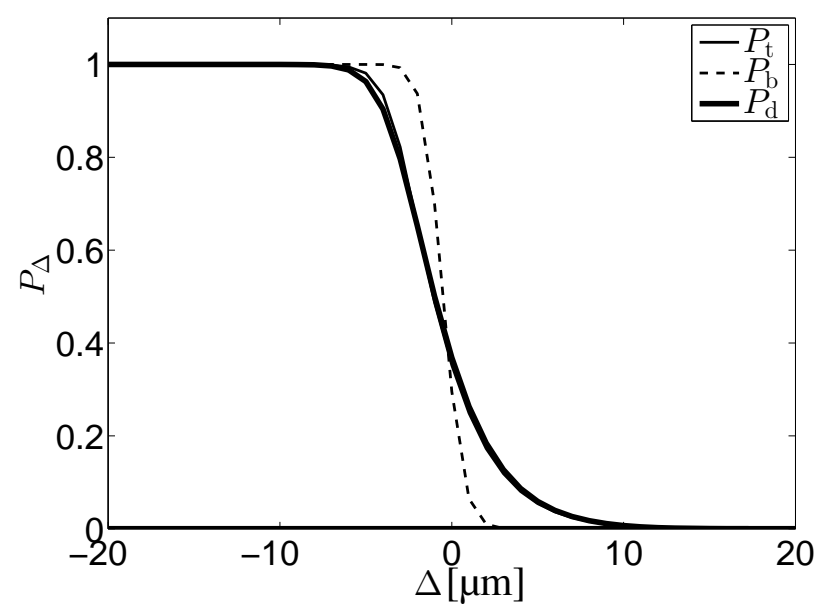

(a)

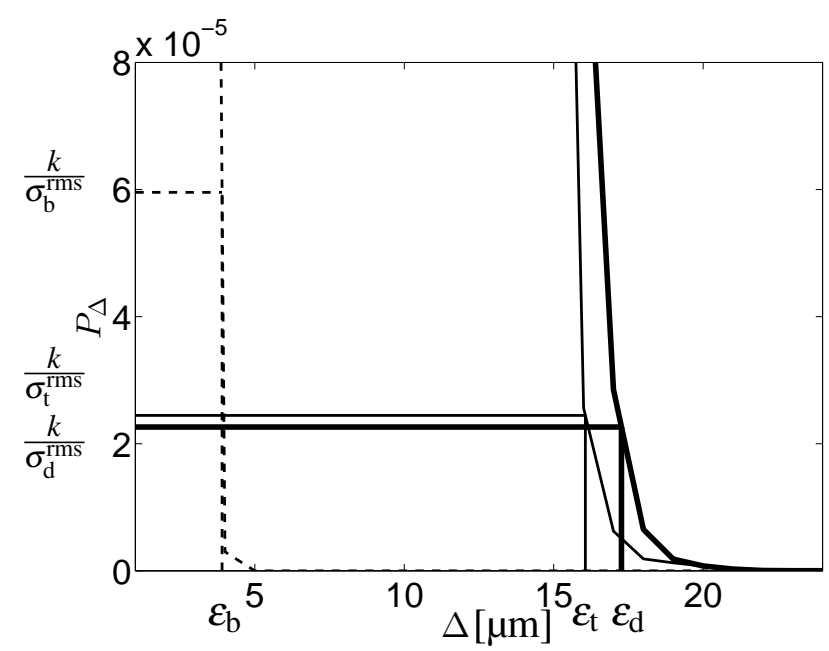

(b)

Figure 4: (a) The complementary cumulative surface height distributions $P_{\mathrm{t}}, P_{\mathrm{b}}$ and $P_{\mathrm{d}}$ for a sample of type T50. (b) Scaled-up subregion of the graphs. Also shown are the values of $k / \sigma_{i}^{\text {rms }}$ and $\varepsilon_{i}$ for $i=\mathrm{t}, \mathrm{b}, \mathrm{d}$, used in experimental calibration.

radiata market kraft pulp fibres [18]. The films also contained varying amounts of NFC pre-treated with TEMPO mediated oxidation, as described by Saito [19]. As reported in [20], the mass fraction of TEMPO-pretreated NFC controls the smoothness of the film. By keeping the grammage of the films constant at $20 \mathrm{~g} / \mathrm{m}^{2}$, films with approximately constant mean thickness but varying roughness were obtained. Details of the manufacturing process and the structural and chemical composition of the films and the precursors may be found in $[3,18,20]$. Total of five sets of films were manufactured, titled T0, T30, T50, T70 and T100, where the numerical part denotes the mass fraction of TEMPO NFC. Consequently, the sets T0 and T100 constitute of the roughest and the smoothest film samples of the series, respectively (see Table 1). The apparent thickness of each film was measured using L \& W Micrometer 51 from ten local areas, and the results were averaged to yield the apparent thickness for each film type (see
Figure 6).

For laser profilometry imaging, a set of samples of $10 \mathrm{~mm} \times 10 \mathrm{~mm}$ were cut from each film and coated with a thin layer of gold. Ten laser profilometry images (of size $1 \mathrm{~mm} \times 1 \mathrm{~mm}$ ) were acquired from each sample surface using a Lehmann laser profilometer (Lehman Mess-Systeme AG, BadenDättwil, Germany). Images with marked artefacts were discarded, and the remaining ones (4-10 for each surface) were filtered as described in Section 3 and binned to obtain the surface height distributions and the root-mean-square surface slope $\sigma^{\mathrm{rms}}$. An example of the resulting complementary cumulative surface height distributions is shown in Figure 4. The experimental values of root-mean-square surface slope are given in Table 1 with uncertainty limits obtained as the standard error of the mean over the replicate samples.

In order to obtain the reference intrinsic thickness $\langle d\rangle_{\mathrm{CT}}$ for the film sets and to calibrate the present 
method using $\mathrm{X}-\mu \mathrm{CT}$, film samples of approximately $1 \mathrm{~mm} \times 4 \mathrm{~mm}$ were cut from each material and glued onto the top of a sample holder rod. Tomographic images of three samples of type $\mathrm{T} 0$ and $\mathrm{T} 30$ and four samples of type T50, T70 and T100 were taken using XRadia $\mu$ CT-400 device (XRadia, Concord, California, USA) with voxel size of $0.58 \mu \mathrm{m}$, see Figure 5. The size of the three-dimensional image was thus approximately $1 \mathrm{~mm} \times 1 \mathrm{~mm} \times 1 \mathrm{~mm}$ with spatial resolution of approximately $1 \mu \mathrm{m}$. The images were binarized using the Otsu method [21], keeping the threshold value constant for all the images. The reference thickness $\langle d\rangle_{\mathrm{CT}}$ was determined for each film type as the mean vertical distance between locations of the upper and lower boundaries between the film material and the ambient air, averaged over the set of samples. The calibration parameter $k$ was determined experimentally based on the value $\langle d\rangle_{\mathrm{CT}}$ for the film type T50, resulting in $k=\left(18_{-9}^{+14}\right) \times 10^{-5}$ for the inflexible case and $k=\left(0.9_{-0.7}^{+2.0}\right) \times 10^{-5}$ for the flexible case. The uncertainty limits were obtained as probabilistically symmetric $68 \%$ coverage intervals, estimated using a Monte Carlo method based on the statistical distribution of all experimental data for $d_{\mathrm{app}}$ and $\langle d\rangle_{\mathrm{CT}}[22]$.

Figure 6a shows the estimated values of intrinsic thickness according to the inflexible model, Equation 10, and the flexible model, Equation 14 with experimental calibration based on samples of film type T50. Also shown are the values of the apparent thickness and the reference intrinsic thickness ob-

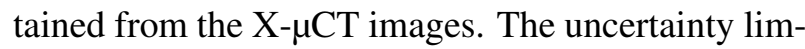
its shown for the intrinsic thickness for the flexible and inflexible cases were obtained using the Monte Carlo method as in the case of calibration parameter discussed above. The uncertainty estimates given for the apparent thickness $d_{\text {app }}$ and the reference thickness $\langle d\rangle_{\mathrm{CT}}$ were obtained as the experimental standard error of the mean over the set of samples mea- sured.

As expected on the basis of approximately the same grammage of the five film types, the measured values of $\langle d\rangle_{\mathrm{CT}}$ are approximately the same for all sample types. Furthermore, the values of apparent thickness $d_{\mathrm{app}}$ are larger than $\langle d\rangle_{\mathrm{CT}}$ for all film types. The deviation between these two values is significant for all but perhaps the smoothest film type T100, being more than a factor of two for the roughest film types. The estimates of intrinsic thickness given both by the flexible and the inflexible models agree well with the reference values $\langle d\rangle_{\mathrm{CT}}$ for film types T0, T30, T70 and T100. The difference between the estimates and the $\mathrm{X}-\mu \mathrm{CT}$ result is largest for the roughest films, but even then the estimated values are much closer to the reference value $\langle d\rangle_{\mathrm{CT}}$ than the measured apparent thickness.

In addition to $\mathrm{X}-\mu \mathrm{CT}$-based calibration, a theoretical estimate of calibration parameter $k$ according to Equation 15 was calculated using the values of Young's modulus $E=11 \pm 1 \mathrm{GPa}$, Poisson's ratio $v=0.3 \pm 0.1[23,24], \kappa=2, F=20 \mathrm{~N}$ and $A_{0}=$ $2 \mathrm{~cm}^{2}$ leading in $k=(1.7 \pm 0.2) \times 10^{-5}$. The resulting estimates of intrinsic thickness together with the apparent and reference thickness values are shown in Figure 6b. In general, the estimates given by the flexible model still agree reasonably well with the $\mathrm{X}-\mu \mathrm{CT}$ results for all film types. The deviation of the intrinsic thickness given by the inflexible model from the reference value $\langle d\rangle_{\mathrm{CT}}$ grows with increasing roughness, and is conspicuous for the film type T0 which also is the most porous one of all the five film types studied here [20]. The flexible model thus appears better suited for the present film samples. Notice that unlike with the experimental calibration, the same value of calibration parameter was used here for both the flexible and the inflexible cases. 


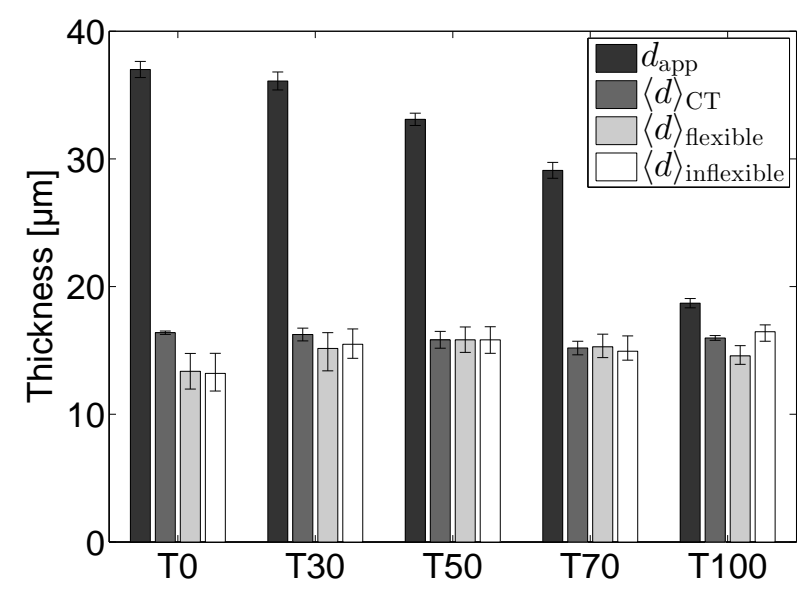

(a) $\mathrm{X}-\mu \mathrm{CT}$ calibration to T50

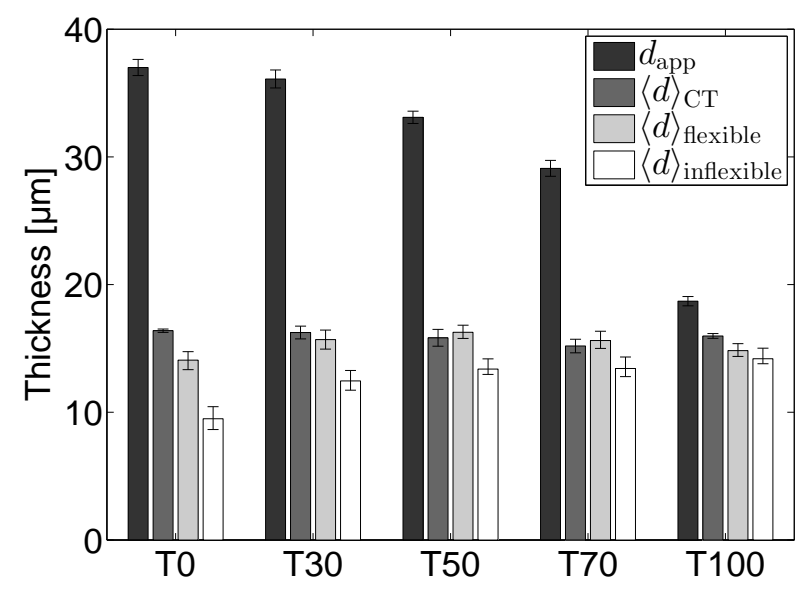

(b) Calibration using Equation 15

Figure 6: Comparison of thickness estimates for NFC films. $d_{\text {app }}$ is the apparent thickness, $\langle d\rangle_{\mathrm{CT}}$ is the intrinsic thickness measured from the X- $\mu \mathrm{CT}$ images, $\langle d\rangle_{\text {inflexible }}$ and $\langle d\rangle_{\text {flexible }}$ are the intrinsic thicknesses obtained using Equations 10 and 14, respectively. In (a) the calibration has been determined from X- $\mu \mathrm{CT}$ data and in (b) from the theory of contact mechanics using Equation 15.

\section{Conclusions}

A method for correcting the (apparent) membrane thickness obtained by a standard micrometric method (ISO534) for membrane surface roughness is introduced, so as to obtain a better estimate of the actual geometrical mean thickness (intrinsic thickness) of a rough membrane. The method can be applied to membranes and sheet materials with no irregular surface structures such as long protruding filaments or lamellae and large surface pores It is based on surface profilometric data and includes a single material-dependent calibration parameter which should be determined experimentally. An alternative method for estimating the calibration parameter based on previous theoretical results is also introduced. The correction depends on the (generally unknown) mechanical behaviour of the membrane in the micrometric thickness measurement. Here, two limiting cases, the inflexible case and the freely flexible case, were considered and found to yield very similar results to each other and to the experimentally determined dataset.

The method was tested on a set of nanofibrillated cellulose film samples representing five different levels of roughness. The surface topology of both sides of the samples was measured using laser profilometry. The value of the calibration parameter was estimated for the test material both theoretically and experimentally. The experimental calibration was obtained by utilizing X-ray microtomographic data from a subset of film samples representing the same intermediate roughness level. The intrinsic thickness estimates thereby obtained were compared with reference values obtained from independent three-dimensional analysis of X-ray micro- 

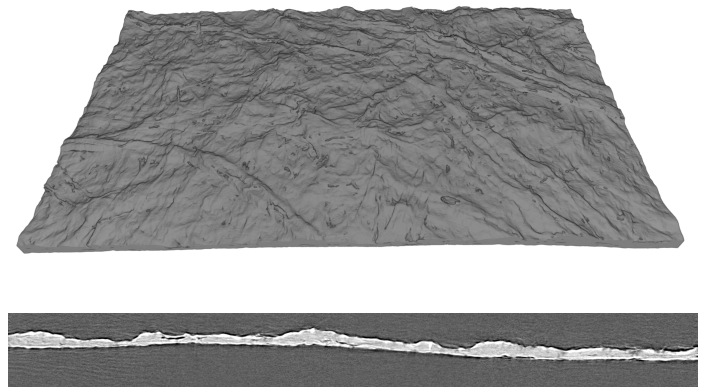

(a)
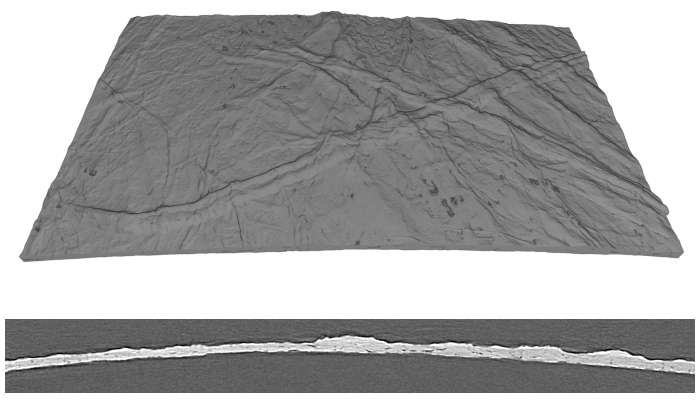

(b)
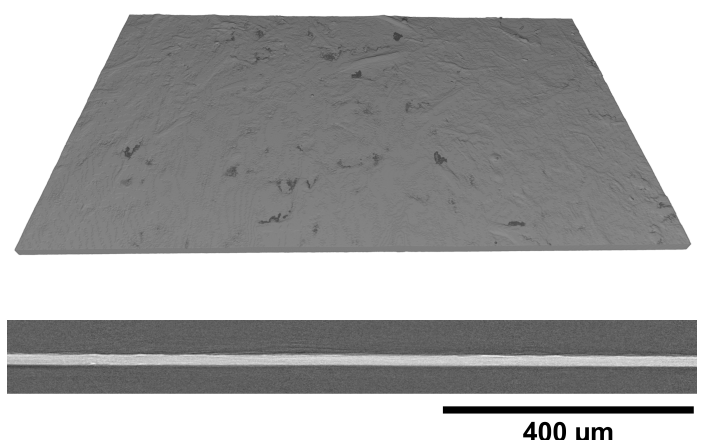

(c)

Figure 5: A portion of size $1.1 \mathrm{~mm} \times 0.7 \mathrm{~mm}$ of the

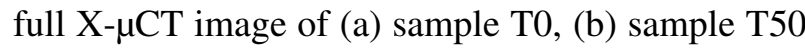
and (c) sample T100. The upper and lower subimages show the perspective view of the top surface and a single cross-sectional slice perpendicular to the membrane plane, respectively. tomographic images.

For the test film set used here, the standard micrometric thickness measurement overestimated the intrinsic thickness, the deviation being roughly a factor of two for all but the smoothest film type. The estimates of intrinsic thickness given both by the flexible and the inflexible models with experimental calibration agree well with the reference values obtained from $\mathrm{X}-\mu \mathrm{CT}$. The difference between the estimates and the reference values is largest for the roughest films $(\sim 15 \%)$, but even in that case the correction provides clear improvement as compared to the deviation of $\sim 230 \%$ of the original apparent thickness value. For the present nanocellulose film materials the estimates of intrinsic thickness obtained appear reasonably accurate for both the experimentally and theoretically calibrated measurements, especially when using the flexible model.

As the method introduced here is based on standard techniques commonly available in many laboratories, a micrometric thickness measurement and surface profilometry, it can provide a practical method for improved estimation of the intrinsic thickness of rough membranes and thereby for more realistic quantification of other important membrane properties such as bulk density, gas barrier properties and various mechanical and optical characteristics.

\section{References}

[1] ISO 534 (2005) Paper and board - Determination of thickness, density and specific volume, International Organization for Standardization, Geneva, Switzerland

[2] Niskanen K, ed. (1998) Paper physics, Papermaking Science and Technology, Fapet, Helsinki, Finland

[3] Chinga-Carrasco G, Yu Y, Diserud O (2011) 
Quantitative electron microscopy of cellulose nanofibril structures from Eucalyptus and Pinus radiata pulp fibres, Microscopy and microanalysis, 17:563-571

[4] Henriksson M, Berglund L (2007) Structure and properties of cellulose nanocomposite films containing melamine formaldehyde, $\mathrm{J}$ Appl Polym Sci, 106:2817-2824

[5] Syverud K, Stenius P (2009) Strength and barrier properties of MFC films, Cellulose, 16:7585

[6] Spence K, Venditti R, Habibi Y, Rojas O, Pawlak J (2010) The effect of chemical composition on microfibrillar cellulose films from wood pulps: Mechanical processing and physical properties, Bioresour Technol, 101:59615968

[7] Rodionova G, Lenes M, Eriksen O, Gregersen O (2010) Surface chemical modification of microfibrillated cellulose: improvement of barrier properties for packaging applications, Cellulose, 18:127-134

[8] Peltonen J, Järn M, Areva S, Linden M, Rosenholm J (2004) Topographical parameters for specifying a three-dimensional surface, Langmuir, 20:9428-9431

[9] Chinga-Carrasco G (2004) Detailed paper surface characterization for gloss assessment, J Pulp Pap Sci, 30:222-227

[10] Järnström J, Ihalainen P, Backfolk K, Peltonen J (2008) Roughness of pigment coatings and its influence on gloss, Appl Surf Sci, 254:57415749
[11] Kak AC, Slaney M (1988) Principles of Computerized Tomographic Imaging, IEEE Press, New York

[12] Hyun S, Robbins M (2007) Elastic contact between rough surfaces: Effect of roughness at large and small wavelengths, Tribol Int, 40:1413-1422

[13] Greenwood JA, Williamson JBP (1966) Contact of nominally flat surfaces, P Roy Soc Lond A Mat, 295:300-319

[14] Bush A, Gibson R, Thomas T (1975) The elastic contact of a rough surface, Wear, 35:87-111

[15] Persson BNJ (2001) Elastoplastic contact between randomly rough surfaces, Phys Rev Lett, 87:116101-1-116101-4

[16] Persson BNJ (2001) Theory of rubber friction and contact mechanics, J Chem Phys, 115:3840-3861

[17] Johnson K (1987) Contact mechanics, Cambridge University Press

[18] Syverud K, Chinga-Carrasco G, Toledo J, Toledo P (2010) A comparative study of Eucalyptus and Pinus radiata pulp fibres as raw materials for production of cellulose nanofibrils, Carbohyd Polym, 84:1033-1038

[19] Saito T, Nishiyama Y, Putaux JL, Vignon M, Isogai A (2006) Homogenous suspensions of individualized microfibrils from TEMPO-catalyzed oxidation of native cellulose, Biomacromolecules, 7:1687-1691

[20] Miettinen A, Chinga-Carrasco G, Kataja M (2014) Three-dimensional microstructural properties of nanofibrillated cellulose films, Int J Mol Sci, 15:6423-6440 
[21] Otsu N (1979) A threshold selection method from gray level histograms, IEEE T Syst Man Cyb, 9:62-66

[22] JCGM (2008) JCGM 101:2008 Evaluation of measurement data - Supplement 1 to the "Guide to the expression of uncertainty in measurement" - Propagation of distributions using a Monte Carlo method, Joint Committee for Guides in Metrology, France

[23] Mikczinski M, Josefsson G, Chinga-Carrasco G, Syverud K, Gamstedt E, Fatikow S (2012) Introducing an in-situ microrobotic approach for assessing the stiffness properties of microfibrillated cellulose films, in: Proceedings of the 2012 International Paper Physics Conference, $67-73$

[24] Mikczinski M, Josefsson G, Chinga-Carrasco G, Gamstedt E, Fatikow S (2014) Nanorobotic testing to assess the stiffness properties of nanopaper, IEEE Trans Robot, 30:115-119 